\title{
Capturing enveloped viruses on affinity grids for downstream cryo-electron microscopy applications
}

\author{
Gabriella Kiss ${ }^{1}$, Xuemin Chen ${ }^{1}$, Melinda A. Brindley ${ }^{2}$, Patricia Campbell ${ }^{3}$, Claudio L. \\ Afonso $^{4}$, Zunlong $\mathrm{Ke}^{5}$, Jens M. Holl ${ }^{1}$, Ricardo C. Guerrero-Ferreira ${ }^{1}$, Lauren A. Byrd- \\ Leotis $^{3}$, John Steel ${ }^{3}$, David A. Steinhauer ${ }^{3}$, Richard K. Plemper ${ }^{1,2}$, Deborah F. Kelly ${ }^{6}$, Paul \\ W. Spearman ${ }^{1}$, and Elizabeth R. Wright ${ }^{1,{ }^{*}}$ \\ ${ }^{1}$ Division of Pediatric Infectious Diseases. Department of Pediatrics. Emory University School of \\ Medicine. Children's Healthcare of Atlanta. Atlanta, GA 30322 \\ ${ }^{2}$ Center for Inflammation, Immunity \& Infection. Georgia State University. Atlanta, GA 30303 \\ ${ }^{3}$ Department of Microbiology and Immunology. Emory University School of Medicine. GA 30322 \\ ${ }^{4}$ USDA, ARS, Southeast Poultry Research Laboratory, Athens, Georgia, USA \\ ${ }^{5}$ School of Biology, Georgia Institute of Technology, Atlanta GA 30332 \\ ${ }^{6}$ Virginia Tech Carilion Research Institute. Roanoke, VA 24016
}

\begin{abstract}
Electron microscopy (EM), cryo-electron microscopy (cryo-EM), and cryo-electron tomography (cryo-ET) are essential techniques used for characterizing basic virus morphology and determining the three-dimensional structure of viruses. Enveloped viruses, which contain an outer lipoprotein coat, constitute the largest group of pathogenic viruses to humans. The purification of enveloped viruses from cell culture presents certain challenges. Specifically, the inclusion of host-membrane derived vesicles, the complete destruction of the viruses, and the disruption of the internal architecture of individual virus particles. Here, we present a strategy for capturing enveloped viruses on affinity grids for use in both conventional EM and cryo-EM/ET applications. We examined the utility of affinity grids for the selective capture of human immunodeficiency virus (HIV) virus-like particles (VLPs), influenza A, and measles virus (MeV). We applied Nickelnitrilotriacetic acid (Ni-NTA) lipid layers in combination with molecular adaptors to selectively adhere the viruses to the affinity grid surface. This further development of the affinity grid method may prove essential for the gentle and selective purification of enveloped viruses directly onto EM grids for ultrastructural analysis.
\end{abstract}

\section{Keywords}

cryo-electron microscopy (cryo-EM); cryo-electron tomography (cryo-ET); affinity grid (AG); enveloped virus

\footnotetext{
*To whom correspondence should be addressed. erwrigh@emory.edu; Tel. (+1) 404-727-4665; Fax (+1) 404-727-9223.
} 


\section{INTRODUCTION}

Enveloped viruses are a significant population of known viruses that are pathogens to both humans and animals. They are covered in a lipoprotein-coat that is derived from the host cell's plasma membrane. Structural studies of enveloped viruses are essential because determining the overall architecture of the virus and common assembly pathways used by viruses may provide important information for antiviral research. Many enveloped viruses maintain a number of well-ordered macromolecular structures contained within the viral envelope, such as the capsid and the nucleocapsid. However, because the process of budding may be a reflection of host cell type, budding mechanism, or length of infection, most enveloped viruses exhibit a characteristic structural irregularity or pleiomorphism. This structural variability can express itself as slight alterations to the size and internal architecture of the virus, such as with members of the Retroviridae family (Briggs et al., 2006a; Briggs et al., 2006b; Butan et al., 2008; Carlson et al., 2008; de Marco et al., 2010; Heymann et al., 2008; Keller et al., 2011; Wright et al., 2007), to significant variations in the size and shape of the virus, as observed with members of the Orthomyxoviridae and Paramyxoviridae families (Calder et al., 2010; Fontana et al., 2012; Harris et al., 2006; Lee, 2010; Liljeroos et al., 2011; Liljeroos et al., 2013; Loney et al., 2009; Yamaguchi et al., 2008). Unfortunately, the structural heterogeneity of viruses may negatively impact the success of viral purification methods used for the production of highly concentrated viral samples, which are essential for cryo-electron microscopy (cryo-EM) and cryo-electron tomography (cryo-ET) studies.

Viruses for ultrastructural studies are produced through the transfection or infection of a permissive cell-type. The growth of the virus is allowed to proceed for a required number of days. Following the incubation period, the cell culture supernatant or a combination of the supernatant and released cells are combined and clarified by low-speed centrifugation. Subsequent steps of purification include the addition of chemicals to induce the precipitation of the virus; ultrafiltration by tangential flow techniques (Wickramasinghe et al., 2005); high-speed centrifugation through a dense-media cushion and/or a dense-media gradient; and a final dialysis or desalting step to remove excess contrast and ice quality distorting media (Gias et al., 2008; Mbiguino and Menezes, 1991; McGinnes et al., 2006). However, each of the purification methods may artificially select for viruses of a specific size, density, or morphology; limit overall virus concentration (titer); alter the ultrastructure of the virus (Sugita et al., 2011); allow for the inclusion of cellular debris; or limit the probability of removing sucrose or other chemicals from the final preparation.

Recently, "Monolayer Purification" and "Affinity Grid" methods were introduced to the EM field in order to combine established His-tagged protein purification techniques directly with EM sample preparation and purification (Kelly et al., 2008a; Kelly et al., 2008b). Briefly, affinity grids are EM grids that have been coated with a lipid layer that contains a large percentage of non-functionalized lipids combined with a variable percentage of lipids that have the Ni-NTA (Nickel-nitrilotriacetic acid) moiety. The Ni-NTA lipid binds directly to either the His-tagged protein of interest or to His-tagged Protein A that is bound to an antibody specific for the target protein. This technique has been successfully applied to the purification and structural analyses of the ribosome and RNA polymerase II from crude cell 
extracts (Kelly et al., 2010b), entire RNA processing pathways (Tanner et al., 2012), and the development of an in situ biological TEM imaging platform (Gilmore et al., 2013). Here, we illustrate how affinity grid technologies may be used to capture pleiomorphic-enveloped viruses directly to EM grids that will be used for conventional TEM or cryo-EM/cryo-ET studies. The application of this technology for cryo-EM studies will provide novel prospects for imaging viruses that have been challenging under conventional methods.

\section{MATERIALS AND METHODS}

\section{Growth and purification of Measles virus}

To generate a recombinant measles virus ( $\mathrm{recMeV}$ ) variant harboring a triple Flag (Zhang et al., 2001) epitope-tagged hemagglutinin (H) protein, site-directed mutagenesis (QuikChange) was employed using a carboxy-terminally single Flag-tagged MeV HEdmonston (Plemper et al., 2001) as template. The resulting H-Edm 3 xFlag-encoding open reading frame (ORF) was sequence confirmed and transferred into a cDNA copy of the $\mathrm{MeV}$-Edm genome, replacing the standard H ORF. Recombinant recMeV-H-Edm 3xFlag virions were recovered as previously described (Plemper et al., 2001), and the presence of the epitope tag confirmed by immunoblot analysis of infected cell lysates using specific antiFlag antibodies (M2; Sigma-Aldrich, St. Louis, MO).

For virus production, ten $15 \mathrm{~cm}$ dishes of Vero-SLAM cells stably expressing human signaling lymphocyte activation molecule (Ono et al., 2001) were infected with recMeV-H$\mathrm{Edm}_{3 x \mathrm{Flag}}$ at a multiplicity of infection (MOI) of 0.005 . Released viral particles were collected when the cells showed more than $90 \%$ syncytia, approximately four days postinfection. Supernatant was clarified of cellular debris through a $0.45 \mu \mathrm{M}$ filter and PEG precipitated by adding $10 \%$ PEG 8000 and $2 \% \mathrm{NaCl}$ to the final volume of the virus containing solution. The virus was then pelleted by centrifugation at $8000 \times \mathrm{g}$ for 1.5 hours at $4^{\circ} \mathrm{C}$ and the virus/PEG pellet gently resuspended in TNE buffer (10mM Tris, $\mathrm{pH} 7.5$; $100 \mathrm{mM} \mathrm{NaCl} ; 1 \mathrm{mM}$ EDTA). Resuspended material was layered on a $20-60 \%$ sucrose cushion, centrifuged at $100,000 \times \mathrm{g}$ for 1.5 hours, and viral particles collected from the interface of the $20-60 \%$ sucrose layers. To reduce the quantity of sucrose in the sample, the recovered virus-containing fraction was diluted in TNE buffer and centrifuged at 100,000 $\times \mathrm{g}$ for 20 minutes. The pellet was then resuspended in TNE and centrifuged for a second time as above. The final pellet was resuspended in $200 \mu \mathrm{L}$ of TNE and yielded titers of $\sim 10^{7}$ $\mathrm{TCID}_{50}$ units/ml.

\section{Growth and purification of Influenza virus}

For the propagation of influenza virus particles, 11 day-old pathogen free embryonated chicken eggs were infected with a 7:1 recombinant virus possessing 7 segments of influenza A/Puerto Rico/34 (H1N1), and A/swine/Spain/53207/04 (H1N1) virus M segment (PR8 7:1). The virus was generated by standard methods (Fodor et al., 1999), and exhibits a pleiomorphic morphology. After incubation for forty-eight hours at $37^{\circ} \mathrm{C}$, the allantoic fluid was harvested and centrifuged at $1,700 \times \mathrm{g}$ for $10 \mathrm{~min}$ at $4^{\circ} \mathrm{C}$ to remove cell debris. The supernatant was then transferred to an ultracentrifuge tube and centrifuged at $12,000 \times \mathrm{g}$ for $30 \mathrm{~min}$ at $4{ }^{\circ} \mathrm{C}$. The clarified supernatant was layered on a $30 \%$ sucrose cushion and 
centrifuged at $77,000 \times \mathrm{g}$ for 1.5 hours at $4^{\circ} \mathrm{C}$. The pellet was resuspended in $100 \mu \mathrm{L}$ of PBS overnight on ice. The sucrose was removed by dialysis for 6 hours against PBS at $4^{\circ} \mathrm{C}$. The final concentrated aliquots yielded titers of $\sim 10^{8}$ to $\sim 10^{10} \mathrm{PFU} / \mathrm{ml}$.

\section{Production and purification of HIV virus-like particles that contain CD84}

PCR fragments of codon-optimized HIV-1 Gag and HIV-1 JRFL Env were introduced sequentially into the plasmids pcDNA4/TO (Invitrogen, Carlsbad CA) and pcDNA5/TO(p) (the plasmid was modified by replacing the Hygromycin gene sequence of pcDNA5/TO with Puromycin gene to make a vector with a puromycin selection marker) to generate plasmid pcDNA4/TO Gag and pcDNA5/TO(p) Env. Expression of both Gag and Env in the plasmids pcDNA4/TO and pcDNA5/TO(p) Env is under a tetracycline-controlled cytomegalovirus (CMV) promoter. First, the pcDNA4/TO Gag was used to transfect a TRex-293 cell line (Invitrogen, Carlsbad CA) that expressed the tetracycline repressor protein TetR. The T-Rex 293 cell line was grown in DMEM containing $5 \mu \mathrm{g} / \mathrm{mL}$ of blasticidin and was transfected using the lipofectmine 2000 method. Forty-eight hours post-transfection, the cells were split into fresh medium containing $5 \mu \mathrm{g} / \mathrm{mL}$ blasticidin and $200 \mu \mathrm{g} / \mathrm{mL}$ zeocin for stable cell line selection. Single foci were selected and expanded. Clones growing in individual wells were induced with doxcycline $(2 \mu \mathrm{g} / \mathrm{mL})$ and the supernatants were harvested at interval time points to test for tetracycline-inducible HIV-1 Gag expression using p24 capture ELISA. Cell clones with the highest Gag expression upon induction and strong doxcycline control were amplified and further characterized. The HIV Gag production cell line was named T-Rex293 Gag. The T-Rex293 Gag cell line was transfected with the plasmid pcDNA5/TO(p) Env using the same method as above. The HIV Gag and Env expression stable cell line was selected using media containing $5 \mu \mathrm{g} / \mathrm{mL}$ blasticidin, 200 $\mu \mathrm{g} / \mathrm{mL}$ zeocin and $1 \mu \mathrm{g} / \mathrm{mL}$ puromycin. HIV-1 Env expression was measured with gp120 capture ELISA. Cell clones with the highest Gag and Env expression upon doxcycline induction and demonstrated complete cleavage of gp160 to gp41 and gp120 were amplified and further characterized. The cell line was named T-Rex293 Gag/Env.

For production of CD84-expressing HIV-1 virus-like particles (VLPs), the human CD84 gene was cloned into pcDNA5/TO vector to generate pcDNA5/TO CD84. PcDNA5/TO CD84 was transfected into a T-Rex293 Gag/Env stable cell line. The transfected cell lines were selected by using $200 \mu \mathrm{g} / \mathrm{mL}$ of hygromycin, $200 \mu \mathrm{g} / \mathrm{mL}$ of zeocin and $1 \mu \mathrm{g} / \mathrm{mL}$ puromycin. The stable cell line after this selection produces HIV-1 gag/Env VLPs with CD84 protein incorporated upon doxycycline induction.

HIV CD84 VLPs were harvested from individual clones after two days of $2 \mu \mathrm{g} / \mathrm{mL}$ doxycycline induction, clarified by low-speed centrifugation, and filtered through a $0.45 \mu \mathrm{m}$ filter. At this point, the HIV VLP-containing solution was purified by one of two methods. In the first approach, the solution was further purified by ultracentrifugation through a $20 \%$ sucrose cushion $\left(100,000 \times \mathrm{g}\right.$ for 2 hours, $\left.4^{\circ} \mathrm{C}\right)$. In the second scheme, the solution was purified and concentrated $5 \times$ using Amicon Utra-15 (100K) centrifugal filters (Millipore Corporation, Billerica, MA) and then further concentrated by ultracentrifugation at $100,000 \times \mathrm{g}$ for 2 hours at $4^{\circ} \mathrm{C}$. Viral pellets from both methods were resuspended in $100 \mu \mathrm{L}$ of PBS. The resuspended HIV CD84 VLPs were used for the imaging experiments. 


\section{Preparation of grids}

Negatively stained grids-400-mesh carbon-formvar coated copper grids (EM Sciences, Hatfield, PA) were glow discharged for 30 seconds. Four $\mu \mathrm{L}$ of the virus sample was applied to the grid and let adsorb for one minute before staining for 30 seconds with either $2 \%$ methylamine tungstate (MAT) or $1 \%$ phosphotungstic acid (PTA).

Negatively stained affinity grids-Affinity grids were prepared as described previously (Kelly et al., 2010a). Briefly, appropriate amounts of active (DGS-NTA(Ni), Avanti Polar Lipids) and filler lipids (DOPC, Avanti Polar Lipids, Alabaster, AL) were mixed and added to the top of $15 \mu \mathrm{L}$ distilled water drops. Lipid monolayers were formed after approximately a one-hour incubation on ice. Non-glow discharged, 400-mesh, carbon-formvar coated copper grids (EM Sciences) were placed carefully on the drops for one minute to allow the affinity lipid monolayer to transfer and adhere. The grids with the affinity lipid monolayer attached were lifted, and transferred to capillary tweezers. Aliquots $(4-\mu \mathrm{L})$ of His-tagged protein A (Abcam, Cambridge, MA) was applied to the monolayer and incubated for one minute. The excess solution was removed using Whatman 1 filter paper. Immediately after blotting, $4 \mu \mathrm{L}$ of virus specific antibodies were applied to the grids for one minute. The surplus solution was removed with filter paper and $4 \mu \mathrm{L}$ of the virus suspension was deposited onto the grid and incubated for two minutes. The excess solution was removed with filter paper. The grids were washed four times to remove unbound particles before the negative stain was applied (2\% methylamine tungstate (MAT) or $1 \%$ phosphotungstic acid (PTA)).

Cryo-EM grids-Quantifoil R1.2/1.3 carbon-copper grids (Quantifoil Micro Tools GmbH, Germany) were glow discharged for 30 seconds. Aliquots (4- $\mu \mathrm{L}$ each) of BSA-treated $10 \mathrm{~nm}$ gold particles (Sigma-Aldrich) were applied to the grids and let air-dry. The virus sample (4$\mu \mathrm{L}$ ) was applied to the grid and allowed to disperse for one minute. The grids were plungefrozen using a FEI Mark III Vitrobot (FEI, Hillsboro, OR).

Cryo-EM affinity grids-Quantifoil R1.2/1.3 carbon-copper grids were treated with ethyl acetate to remove plastic film residues. The monolayer was attached to the copper back-side of the grid for greater stability. Solutions were applied to the monolayer as described for the negatively stained affinity grids, excess solutions were pipetted away, no wash steps were performed. Virus sample (4- $\mu \mathrm{L})$ was added to the grid and allowed to attach for one minute. A second aliquot of the virus specimen was mixed in a 1:1 ratio with the BSA-treated $10 \mathrm{~nm}$ gold particles in a total volume of $2.5 \mu \mathrm{L}$ and applied to the grid. The grids were plungefrozen using a FEI Mark III Vitrobot.

\section{Electron microscopy}

Negatively stained specimens were imaged on a JEOL JEM-1400 transmission electron microscope (TEM; JEOL, Ltd., Japan) equipped with a $\mathrm{LaB}_{6}$ filament and operated at an accelerating voltage of $120 \mathrm{kV}$. Images with magnifications of 1,500×, 4,000×, and 12,000× were digitally captured on a Gatan US1000 $(2 \mathrm{k} \times 2 \mathrm{k})$ CCD camera (Gatan, Pleasanton, CA). 
Vitrified specimens were either imaged on the JEOL JEM-1400 $120 \mathrm{kV}$ TEM, as described above, or the JEOL JEM-2200FS, $200 \mathrm{kV}$ FEG-TEM with an in-column Omega energy filter operated at a slit width of $20 \mathrm{eV}$. Images on the JEOL JEM-2200FS were collected with a Gatan US4000 $(4 \mathrm{k} \times 4 \mathrm{k}) \mathrm{CCD}$ camera. Images were automatically binned by two and acquired with a pixel size equal or less than 7.6 $\AA$. For individual images, a total electron dose of $\sim 5 \mathrm{e}-/ \AA^{2}$ was used. For tilt series acquisition, a cumulative electron dose of $\sim 120$ $\mathrm{e}-/ \AA^{2}$ was distributed over tilt series ranging from $-62^{\circ}$ to $+62^{\circ}$. Images were acquired at $-4.0 \mu \mathrm{m}$ defocus (first CTF zero: $0.31 \mathrm{~nm}^{-1}$ ). Tilt series images were automatically collected with $2^{\circ}$ of angular increments using the predictive SerialEM package (Mastronarde, 2005).

\section{Image processing}

Images from the negatively stained samples were used to quantify the number of viral particles present under each affinity grid preparation method. Three-dimensional (3D) reconstructions (tomograms) were generated from the aligned image stacks using IMOD Version 4.3.1 (Kremer et al., 1996).

\section{Virus and particle quantification}

All particles in the field of view were counted in five 12kx images acquired from each preparative condition for both the negatively stained grids and cryo-EM grids. For the unambiguous quantification of $\mathrm{MeV}$ on untreated cryo-grids and cryo- $\mathrm{AG}$, all particles present were counted in five 3D reconstructions that were collected at a magnification of $30 \mathrm{kx}$. Graphs represent the mean particle count from the five replicates; the standard deviations were calculated in Microsoft Office Excel. Particles were categorized as 'viruses' if they displayed the characteristic viral morphology that is known for each of the three viruses studied. In brief, a particle was classified as a virus if it maintained the general size and shape of each of the three viruses; had an intact viral envelope decorated with the virusspecific surface glycoproteins; and contained internal structural complexes consistent with each virus, such as the ribonucleoprotein (RNP) complex for $\mathrm{MeV}$ and influenza and the Gag lattice for the HIV VLPs. Particles not meeting the above criteria were categorized as 'other particles.'

\section{RESULTS AND DISCUSSION}

\section{A. Paramyxoviruses}

Paramyxoviruses are significant human pathogens known for their extreme pleomorphicity and difficult purification. Viruses from the Paramyxoviridae family display a broad range of shapes and sizes, which have lead to significant controversies regarding whether all the morphologies reported represent true viruses or if some may be defective interfering particles (Hall et al., 1974; Maeda et al., 1978; Valdovinos and Gomez, 2003). A major hindrance to resolving this debate is the inability to obtain highly concentrated and pure viral samples for conventional TEM or cryo-EM structural studies. Therefore, the optimization of AG virus purification will be a practical solution for improving the success of EM-based studies of paramyxoviruses. 
$\mathrm{MeV}-\mathrm{MeV}$ displays the greatest degree of structural pleomorphicity of all the viruses examined by our group. In addition, the majority of $\mathrm{MeV}$ progeny particles remain cellassociated, which increases the possibility of the incorporation of vesicles and other cellular debris decorated with the viral glycoproteins into the 'purified' virus preparation. Both of these attributes make cryo-EM structural analyses of MeV extremely challenging. In our study, we used the affinity grid (AG) techniques for $\mathrm{MeV}$ in order to increase the number of virus particles adsorbed to the grid surface while simultaneously reducing the quantity of other contaminants associated with the grid so that the virus sample would be more feasible for ultrastructural analysis. RecMeV-H-Edm $3 \times$ FLAG was engineered to display the Flag epitope-tag at a membrane-distal position of the $\mathrm{H}$ protein head domain. The inclusion of the Flag-tag does not impact virus infectivity (Plemper et al., 2001) or morphology (Figure 1A and $1 \mathrm{~B}$; Figure $2 \mathrm{C}$ and $2 \mathrm{D}$ ). The addition of the Flag-tag made the virus useful for affinity purification.

Using a high affinity anti-Flag antibody, particles were successfully recruited to the AG (Figure 1B). Due to the unavoidable presence of glycoprotein-studded vesicles in the preparation, a mixture of $\mathrm{MeV}$ particles and vesicles did adhere to the AG. However, there was a significant decrease in the cellular debris localized to the AG surfaces when compared with the conventional EM grids (Figure 1A). This indicated that only particles, which had viral glycoproteins on their surface, were able to bind to and remain associated with the AG.

We quantified the number of $\mathrm{MeV}$-Flag particles and other particles present on untreated EM grids and on the AG to further confirm the utility of the AG technique when used to capture MeV-Flag particles on EM grids (Figure 1C; Supplementary Table 1). Our analysis revealed that there were, on average, greater numbers of non-viral particles $(12.0 \pm 4.85)$ present versus the MeV-Flag particles $(8.8 \pm 3.19)$ on the untreated EM grids. However, on the AG, while there were fewer particles in total, the quantity of identifiable MeV-Flag particles was greater when compared to the amount of other particles on the grids. We determined that when the amount of the Ni-NTA lipid was increased in the affinity monolayer the recruitment of the MeV-Flag particles was enhanced, as was measured for $2 \%(3.0 \pm 1.73), 20 \%(5.4 \pm 2.0)$, and 50\% (9.6 \pm 3.6$)$ Ni-NTA lipid. Interestingly, the amount of the other particles present on the AG surface was low, with the mean number of particles remaining at $\sim 3$ (Figure 1C; Supplementary Table 1). We did observe that the proportionality between the percentage of the Ni-NTA lipid in the monolayer and MeV-Flag particle recruitment was not as pronounced as it was for the influenza particles (Figure 3; Supplementary Table 3) or the HIV VLPs (Figure 5; Supplementary Figure 2; and Supplementary Table 5), this could be attributed to several points. First, the significant structural heterogeneity of the MeV-Flag particles could have impacted the orientation and exposure of the H-Flag epitope-tag to the AG surface and thereby limited the viral particles' degree of association with the grid surface. Second, the lower titer of the MeV-Flag particles $\left(\sim 10^{7} \mathrm{TCID}_{50}\right.$ units $\left./ \mathrm{ml}\right)$ combined with the higher quantity of background material when compared to that of either the highly pure and concentrated influenza particles $\left(\sim 10^{8-10}\right.$ PFU/mL) or HIV VLPs could have limited the adherence of MeV-Flag particles to the AG. We determined that removing the incorporation of the antibody from the AG experiment resulted in the loss of MeV-Flag particle attachment to the AG. We were able to elute MeV- 
Flag particles from the $\mathrm{AG}$ with the addition of $600 \mathrm{mM}$ imidazole. Both attributes suggest that the interaction between MeV-Flag particles and the monolayer was maintained by the antibody-protein A-His-Ni-NTA complex (Figure 1C; Supplementary Table 1). Subsequent to treatment of the AG with imidazole, we examined if the AG surface would continue to support the recruitment of MeV-Flag particles. On the $20 \%$ Ni-NTA lipid containing monolayers, MeV-Flag particles were still able to attach $(1.8 \pm 1.1)$ and the number of nonviral particles remained low $(2.8 \pm 3.6)$. This suggested that the lipid monolayer remained intact and the preferential binding activity supported by the $20 \%$ Ni-NTA AG was stable.

Applying the AG technique to cryo-grids resulted in the presence of fewer particles of all types on the grids (Figure 2B and 2D; Supplementary Table 2), and of those particles present, there were greater numbers of particles with surface glycoproteins and a visible ribonucleoprotein (RNP) complex in the virus interior. This suggests that large fractions of cellular debris and vesicles were removed from the cryo-AG. Cryo-ET data was collected on $\mathrm{MeV}$ particles on both regular cryo-grids (Figure 2C) and cryo-AG (Figure 2D) to determine whether the presence of the monolayer would impact individual image quality and subsequent achievable resolution of the three-dimensional reconstructions. From both grid types, after the generation of the reconstructions, we identified individual glycoproteins on the surface of the virions (Figure 2, white arrowheads), the viral membrane, and the RNP structure inside of the particles (Figure 2, black arrowheads). This indicated that the addition of the lipid layer or the molecular adaptors did not decrease signal from the viral macromolecules or introduce noise to the images. The RNP structure was double-layered, which suggested that the internal layer represents the RNP and the viral matrix protein may form a shell encasing the RNP. This finding supports the previous reports of $\mathrm{MeV}$ generating a combined RNP-matrix complex (Liljeroos et al., 2011).

\section{B. Orthomyxoviruses}

The Orthomyxoviridae family of viruses includes the human pathogens Influenza A, B and C. Viruses from this family exhibit a broad range of morphologies that encompass the small and mostly spherical to the long and filamentous varieties. Orthomyxoviridae viruses are ideal candidates to test the applicability of AG for capturing viruses with lesser pleomorphic character than $\mathrm{MeV}$, but greater morphological heterogeneity than the HIV VLPs. Influenza A/PR8 7:1 [H1N1] virus was used in the AG experiment because preparations of this strain contain a mixture of both spherical and filamentous virus particles (Figure 3). Similar to both MeV and the HIV CD84 VLPs, influenza A/PR8 7:1 particles exhibited a dependency on attachment to the AG when the percentage of the Ni-NTA was varied in the monolayer from $2 \%$ to $50 \%$ (Figure 4B and 4D-4E; Supplementary Table 3).

We assessed if elimination of the Protein A-His or the antibody resulted in a clear decrease or absence of captured influenza A/PR8 7:1 particles, the removal of either component abolished viral particle adherence (Supplementary Figure 1A and 1B; Supplementary Table 3). At the same time, we also determined that influenza A/PR8 7:1 particles were readily eluted from the AG with the addition of $600 \mathrm{mM}$ imidazole (Supplementary Figure 1C). After imidazole treatment, the monolayer remained functional because influenza A/PR8 7:1 particles were able to reattach to the AG monolayer surface (Supplementary Figure 1D; 
Supplementary Table 3). The level of influenza A/PR8 7:1 particle attachment was reduced after imidazole treatment $(26.8 \pm 8.11)$ when compared to the $20 \%$ AG values $(63.0 \pm 8.72)$. This reduction could be due to chemical or physical disruption of the lipid monolayer and the Ni-NTA active groups. Using AG to capture influenza A /PR8 7:1 particles onto cryoEM grids (Figure 4D and 4E, Supplementary Table 4) did not produce as significant an increase in the numbers of captured viral particle as it did for HIV VLPs (Supplementary Table 6). However, there was a greater dispersion and even distribution of the viral particles on the cryo-AG when compared to standard cryo-EM grids (Figure 4A and 4B), which is still advantageous for cryo-EM studies (Figure 4D and 4E).

\section{HIV VLPs}

HIV belongs to the Retroviridae family and is a modestly pleomorphic enveloped virus. We selected the HIV CD84 VLPs for the development of the affinity purification technique because the VLP morphology was relatively uniform; the Env glycoprotein or the CD84 moiety could be selectively targeted for binding; and high affinity anti-Env (provided by Dr. Jason Hammonds) or CD84 (Santa Cruz Biotechnology, Dallas, TX) antibodies were available. The VLPs were concentrated as described, then negatively stained and imaged (Figure 5A; Supplementary Figure 2A). The concentrated HIV VLP stock yielded images of intact, but densely packed VLPs on standard EM grids (Figure 5A; Supplementary Figure 2A). Solutions of the same HIV VLP stocks were then applied to the AG using the antibody/ Protein A-His/Ni-NTA method. As with the other viral systems, we examined if virus attachment could be selectively regulated by increasing the amount of the Ni-NTA lipid in the affinity monolayer from $2 \%$ to $20 \%$ to $50 \%$. When using either the anti-Env antibody or the anti-CD84 antibody, the amount of HIV VLPs on the AG, was dependent on the percentage of Ni-NTA lipid in the monolayer (anti-Env: Figure 5B and 5D - 5E; anti-CD84: Supplementary Figure 2B and 2D - 2E; Supplementary Table 5). Removal of either the Protein A-His or the antibody resulted in insignificant quantities of the HIV VLPs attached to the AG (Supplementary Figure 3A and 3B; Supplementary Table 5). We applied a solution of $600 \mathrm{mM}$ imidazole to the previously imaged AG and successfully eluted the HIV VLPs from the AG (Supplementary Figure 3C; Supplementary Table 5) while retaining the intact lipid monolayer on the grid. Subsequently, fresh aliquots of HIV VLPs reattached to the AG monolayer at levels close to what was observed with first-round $20 \%$ AG (Supplementary Figure 3D; Supplementary Table 5).

We determined whether affinity purification techniques would be of benefit for cryo-EM studies of HIV VLPs. The use of the affinity monolayer on the cryo-EM grid resulted in more even distribution of the HIV VLPs across the entire grid as well as an overall increase in the number of HIV VLPs (Figure 6D and 6E; Supplementary Table 6) as compared to a standard cryo-EM grid (Figure 6A and 6B; Supplementary Table 6). This finding is of significant value for cryo-EM/cryo-ET studies of HIV and HIV VLPs due to the fact that the virus and VLPs have a 'spherical' shape and a diameter of $\sim 125 \mathrm{~nm}$, which tends to encourage them to aggregate and cluster near the edges of the Quantifoil grid holes (Figure 6B). As with MeV-Flag, cryo-ET data was collected of HIV VLPs on both regular cryogrids and cryo-AG to determine whether the presence of the monolayer would impact individual image quality and subsequent achievable resolution of the three-dimensional (3D) 
reconstructions. In the 3D reconstructions, we were able to resolve the Gag polyprotein shells that are a structural hallmark of immature HIV VLPs or immature HIV virions (data not shown). This information showed that neither the AG lipid layer nor the molecular adaptors reduced the quality of the structural information obtainable from the HIV VLPs.

\section{CONCLUSIONS}

Structural studies are fundamental for the development of antivirals that target many families of enveloped viruses. Valuable information is found at both high- and intermediateresolutions, specifically how individual macromolecular interactions contribute to dramatic alterations of overall viral organization or subtle changes such as exposure of specific epitopes. Enveloped viruses exhibit great structural heterogeneity; therefore the best methods for the analysis of intact viral particles are cryo-EM and cryo-ET. In this work, a recent technology known as the "affinity grid" was optimized for the capture of enveloped viruses directly onto EM grids. Prior to the initiation of these studies, three additional experimental factors were considered. First, the availability of a high-affinity antibody, which targets a well-exposed epitope on each of the viruses, was required for the success of the technique. Second, the quantity of the antibody used needed optimization. Finally, we had to either avoid or limit the use of sucrose, detergents, or harsh chemicals during specimen preparation because minute quantities of these compounds would destroy the lipid monolayer.

Viruses from three enveloped virus families, Retroviridae, Orthomyxoviridae, and Paramyxoviridae were chosen because: 1) the families contain a number of highly infectious pathogens to humans and animals, and 2) they represent the significant range of structural heterogeneity present amongst viruses. The individual viruses studied were: HIV CD84 VLPs, influenza A, and MeV. We demonstrated that the AG could selectively capture all of the viruses; the antibody-Protein A-His-Ni-NTA complex maintained the interaction with the particles; and this interaction was strong enough to maintain the coordination of the viruses to the cryo-AG. No preferred virus morphology was observed when the AG methods were employed. However, it was noted that a greater degree of pleiomorphism of the virus apparently coincides with overall lower capturing efficiency of the AG. That said, for all the enveloped viruses examined, a noticeable improvement was observed either in viral particle distribution and/or viral particle numbers on both the negatively stained and cryo-EM AGs. We also found that cryo-ET tilt series collected of viruses on cryo-AGs are suitable for further high-resolution structural studies and that the additional lipid layer does not diminish the valuable signal from the sample or produce noticeable background noise.

In this study, we were not explicitly concerned with possible conformational changes of the surface glycoprotein structures upon their binding with the antibody. If the aim is to determine the native structure of the surface glycoproteins, a promising substitute to conventional antibody-to-glycoprotein interactions may be to employ alternative anchors for the affinity immobilization. For example, in our study of the HIV CD84 VLPs, two binding sites were available, HIV Env and a cellular glycoprotein, CD84. In both cases, we observed selective capture of the HIV VLPs to the AG, but we could be assured that when using the CD84 antibody capture, the structure of HIV Env would not be altered (Supplementary 
Figure 2). The other approach is to engineer viruses with extended His-tags anchored to the surface glycoproteins. To this end, we examined two His-tagged $\mathrm{MeV}$ mutants in which the His-tags were placed on one of the surface glycoproteins. Unfortunately, our results were not consistent, suggesting that there may be other factors that limit its applicability at this time including possible steric-hindrances' from interactions between the two surface glycoproteins or flexibility from the extended architecture of the His-tagged glycoprotein (data not shown). We continue to develop the affinity grid method to improve the success of virus capture without disrupting the lipid monolayer or the structure of the viral glycoproteins. Due to the significant impact that employing affinity grid techniques will afford to the purification of enveloped viruses directly to EM grids, we propose this method as a powerful future tool for ultrastructural analyses of viruses.

\section{Supplementary Material}

Refer to Web version on PubMed Central for supplementary material.

\section{Acknowledgments}

The authors thank Dr. Leon De Masi for providing reagents and support during the preparation of the manuscript. We would like to thank Dr. Jason Hammonds for the anti-Env polyclonal antibody and Dr. Philip Santangelo for the anti-NDV polyclonal antibody. We also thank Ms. Jeannette Taylor and Ms. Hong Yi of the Emory University Robert P. Apkarian Integrated Electron Microscopy Core for assistance. This work was supported in part by Emory University, Children's Healthcare of Atlanta, the Center for AIDS Research at Emory University (P30 AI050409), and the Georgia Research Alliance to E.R.W.; NSF grant 0923395 to E.R.W; and public health service grants AI101775 to E.R.W, AI058828 to P.W.S., and AI083402 to R.K.P. from the NIH/NIAID; USDA_ARS CRIS project number 66612-32000-064 to C.L.A.; and Centers for Excellence in Influenza Research and Surveillance (CEIRS) contract number HHSN266200700006C to J.S. and D.A.S.

\section{References}

Briggs JA, Grunewald K, Glass B, Forster F, Krausslich HG, Fuller SD. The mechanism of HIV-1 core assembly: insights from three-dimensional reconstructions of authentic virions. Structure. 2006a; 14:15-20. [PubMed: 16407061]

Briggs JA, Johnson MC, Simon MN, Fuller SD, Vogt VM. Cryo-electron microscopy reveals conserved and divergent features of gag packing in immature particles of Rous sarcoma virus and human immunodeficiency virus. Journal of molecular biology. 2006b; 355:157-168. [PubMed: 16289202]

Butan C, Winkler DC, Heymann JB, Craven RC, Steven AC. RSV capsid polymorphism correlates with polymerization efficiency and envelope glycoprotein content: implications that nucleation controls morphogenesis. Journal of molecular biology. 2008; 376:1168-1181. [PubMed: 18206161]

Calder LJ, Wasilewski S, Berriman JA, Rosenthal PB. Structural organization of a filamentous influenza A virus. Proceedings of the National Academy of Sciences of the United States of America. 2010; 107:10685-10690. [PubMed: 20498070]

Carlson LA, Briggs JA, Glass B, Riches JD, Simon MN, Johnson MC, Muller B, Grunewald K, Krausslich HG. Three-dimensional analysis of budding sites and released virus suggests a revised model for HIV-1 morphogenesis. Cell Host Microbe. 2008; 4:592-599. [PubMed: 19064259]

de Marco A, Davey NE, Ulbrich P, Phillips JM, Lux V, Riches JD, Fuzik T, Ruml T, Krausslich HG, Vogt VM, et al. Conserved and variable features of Gag structure and arrangement in immature retrovirus particles. Journal of virology. 2010; 84:11729-11736. [PubMed: 20810738]

Fodor E, Devenish L, Engelhardt OG, Palese P, Brownlee GG, Garcia-Sastre A. Rescue of influenza A virus from recombinant DNA. Journal of Virology. 1999; 73:9679-9682. [PubMed: 10516084] 
Fontana J, Cardone G, Heymann JB, Winkler DC, Steven AC. Structural changes in Influenza virus at low pH characterized by cryo-electron tomography. Journal of Virology. 2012; 86:2919-2929. [PubMed: 22258245]

Gias E, Nielsen SU, Morgan LA, Toms GL. Purification of human respiratory syncytial virus by ultracentrifugation in iodixanol density gradient. J Virol Methods. 2008; 147:328-332. [PubMed: 18029032]

Gilmore BL, Showalter SP, Dukes MJ, Tanner JR, Demmert AC, McDonald SM, Kelly DF. Visualizing viral assemblies in a nanoscale biosphere. Lab on a chip. 2013; 13:216-219. [PubMed: 23208001]

Hall WW, Martin SJ, Gould E. Defective interfering particles produced during the replication of measles virus. Medical microbiology and immunology. 1974; 160:155-164. [PubMed: 4216759]

Harris A, Cardone G, Winkler DC, Heymann JB, Brecher M, White JM, Steven AC. Influenza virus pleiomorphy characterized by cryoelectron tomography. Proceedings of the National Academy of Sciences of the United States of America. 2006; 103:19123-19127. [PubMed: 17146053]

Heymann JB, Butan C, Winkler DC, Craven RC, Steven AC. Irregular and Semi-Regular Polyhedral Models for Rous Sarcoma Virus Cores. Comput Math Methods Med. 2008; 9:197-210. [PubMed: 19122884]

Keller PW, Adamson CS, Heymann JB, Freed EO, Steven AC. HIV-1 maturation inhibitor bevirimat stabilizes the immature Gag lattice. Journal of virology. 2011; 85:1420-1428. [PubMed: 21106735]

Kelly DF, Abeyrathne PD, Dukovski D, Walz T. The Affinity Grid: a pre-fabricated EM grid for monolayer purification. Journal of Molecular Biology. 2008a; 382:423-433. [PubMed: 18655791]

Kelly DF, Dukovski D, Walz T. Monolayer purification: a rapid method for isolating protein complexes for single-particle electron microscopy. Proceedings of the National Academy of Sciences of the United States of America. 2008b; 105:4703-4708. [PubMed: 18347330]

Kelly DF, Dukovski D, Walz T. A practical guide to the use of monolayer purification and affinity grids. Methods in enzymology. 2010a; 481:83-107. [PubMed: 20887854]

Kelly DF, Dukovski D, Walz T. Strategy for the use of affinity grids to prepare non-His-tagged macromolecular complexes for single-particle electron microscopy. Journal of Molecular Biology. 2010b; 400:675-681. [PubMed: 20562026]

Kremer JR, Mastronarde DN, McIntosh JR. Computer visualization of three-dimensional image data using IMOD. J Struct Biol. 1996; 116:71-76. [PubMed: 8742726]

Lee KK. Architecture of a nascent viral fusion pore. The EMBO journal. 2010; 29:1299-1311. [PubMed: 20168302]

Liljeroos, L.; Huiskonen, JT.; Ora, A.; Susi, P.; Butcher, SJ. Electron cryotomography of measles virus reveals how matrix protein coats the ribonucleocapsid within intact virions. Proceedings of the National Academy of Sciences of the United States of America; 2011.

Liljeroos L, Krzyzaniak MA, Helenius A, Butcher SJ. Architecture of respiratory syncytial virus revealed by electron cryotomography. Proceedings of the National Academy of Sciences of the United States of America. 2013; 110:11133-11138. [PubMed: 23776214]

Loney C, Mottet-Osman G, Roux L, Bhella D. Paramyxovirus ultrastructure and genome packaging: cryo-electron tomography of sendai virus. Journal of virology. 2009; 83:8191-8197. [PubMed: 19493999]

Maeda A, Suzuki Y, Matsumoto M. Isolation and characterization of defective interfering particle of Newcastle disease virus. Microbiology and immunology. 1978; 22:775-784. [PubMed: 752096]

Mastronarde DN. Automated electron microscope tomography using robust prediction of specimen movements. J Struct Biol. 2005; 152:36-51. [PubMed: 16182563]

Mbiguino A, Menezes J. Purification of human respiratory syncytial virus: superiority of sucrose gradient over percoll, renografin, and metrizamide gradients. J Virol Methods. 1991; 31:161-170. [PubMed: 1650782]

McGinnes LW, Pantua H, Reitter J, Morrison TG. Newcastle disease virus: propagation, quantification, and storage. Current protocols in microbiology. 2006; Chapter 15(Unit 15F): 12 . 
Ono N, Tatsuo H, Hidaka Y, Aoki T, Minagawa H, Yanagi Y. Measles viruses on throat swabs from measles patients use signaling lymphocytic activation molecule (CDw150) but not CD46 as a cellular receptor. Journal of Virology. 2001; 75:4399-4401. [PubMed: 11287589]

Plemper RK, Hammond AL, Cattaneo R. Measles virus envelope glycoproteins hetero-oligomerize in the endoplasmic reticulum. The Journal of biological chemistry. 2001; 276:44239-44246. [PubMed: 11535597]

Sugita Y, Noda T, Sagara H, Kawaoka Y. Ultracentrifugation deforms unfixed influenza A virions. J Gen Virol. 2011; 92:2485-2493. [PubMed: 21795472]

Tanner JR, Degen K, Gilmore BL, Kelly DF. Capturing RNA-dependent pathways for cryo-EM analysis. Computational and Structural Biotechnology Journal. 2012; 1

Valdovinos MR, Gomez B. Establishment of respiratory syncytial virus persistence in cell lines: association with defective interfering particles. Intervirology. 2003; 46:190-198. [PubMed: 12867758]

Wickramasinghe SR, Kalbfuss B, Zimmermann A, Thom V, Reichl U. Tangential flow microfiltration and ultrafiltration for human influenza A virus concentration and purification. Biotechnol Bioeng. 2005; 92:199-208. [PubMed: 16041807]

Wright ER, Schooler JB, Ding HJ, Kieffer C, Fillmore C, Sundquist WI, Jensen GJ. Electron cryotomography of immature HIV-1 virions reveals the structure of the CA and SP1 Gag shells. EMBO J. 2007; 26:2218-2226. [PubMed: 17396149]

Yamaguchi M, Danev R, Nishiyama K, Sugawara K, Nagayama K. Zernike phase contrast electron microscopy of ice-embedded influenza A virus. Journal of structural biology. 2008; 162:271-276. [PubMed: 18313941]

Zhang L, Hernan R, Brizzard B. Multiple tandem epitope tagging for enhanced detection of protein expressed in mammalian cells. Mol Biotechnol. 2001; 19:313-321. [PubMed: 11721627] 

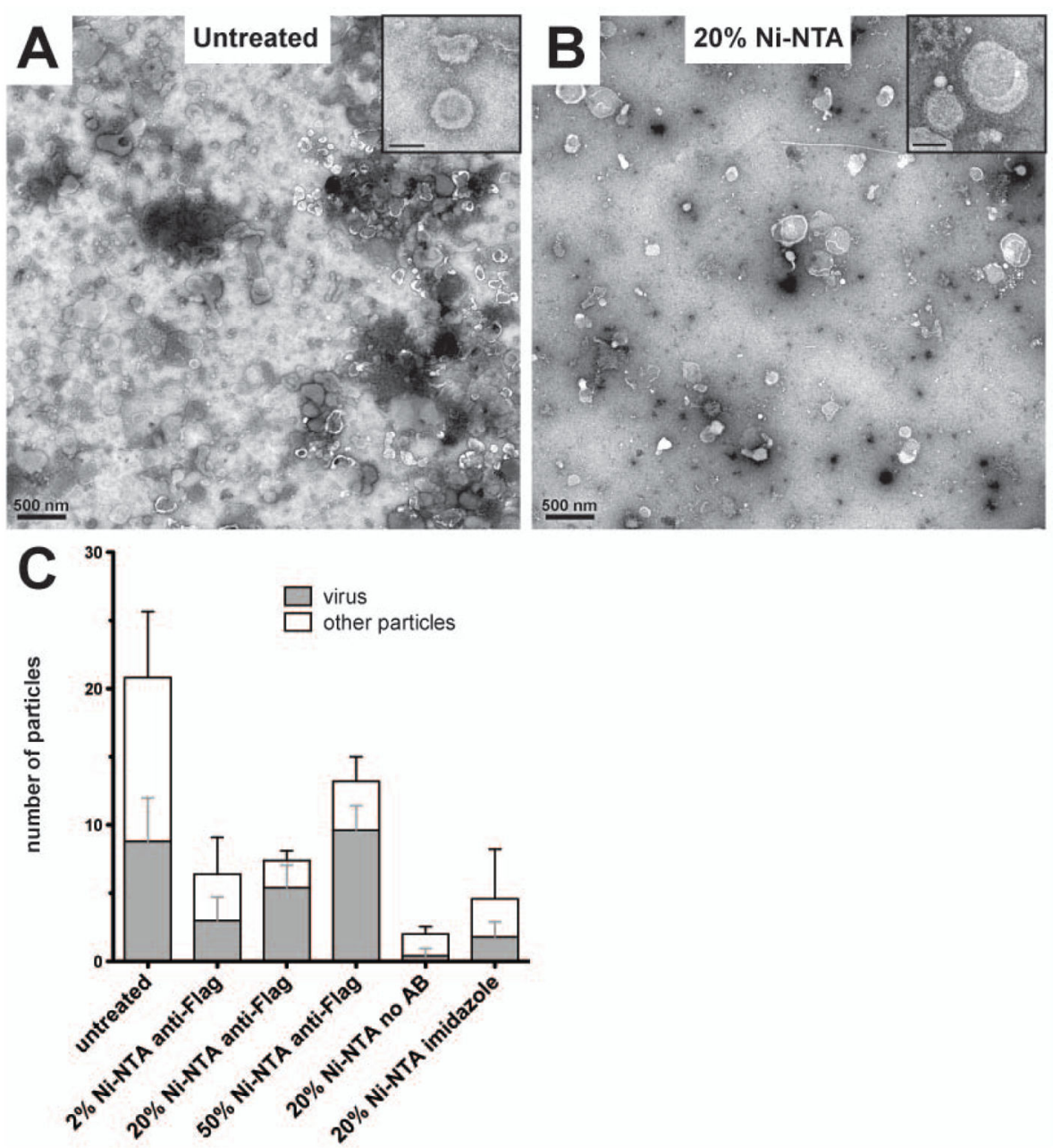

Figure 1.

TEM images of negatively stained recMeV-H-Edm $3 \times$ Flag particles. (A) recMeV-H-

$\mathrm{Edm}_{3 \mathrm{xFlag}}$ particles from the resuspended pellet. (B) recMeV-H-Edm $3 \times$ Flag particles captured on $20 \%$ Ni-NTA containing affinity grid using an anti-Flag antibody (1:500). (C) Quantification of the number of recMeV-H-Edm $3 \times$ Flag (grey) and other (white) particles present on untreated and AG EM grids. Untreated EM grids adsorbed more total particles than the three AGs. The AGs recruited recMeV-H-Edm3xFlag particles in a stepwise-fashion that was proportional to the percentage of the Ni-NTA present in the lipid monolayer. The quantity of other particles present on all the AGs was greatly reduced. AG prepared without the antibody did not recruit particles to the grid surface. Treatment of the AG with $600 \mathrm{mM}$ imidazole resulted in the elution of particles previously bound to the AG surface. Images (A and $\mathrm{B}$ ) were acquired at $4 \mathrm{kx}$ and $12 \mathrm{kx}$ (insets) magnifications. Scale bars are $500 \mathrm{~nm}$ and $100 \mathrm{~nm}$ (insets). 

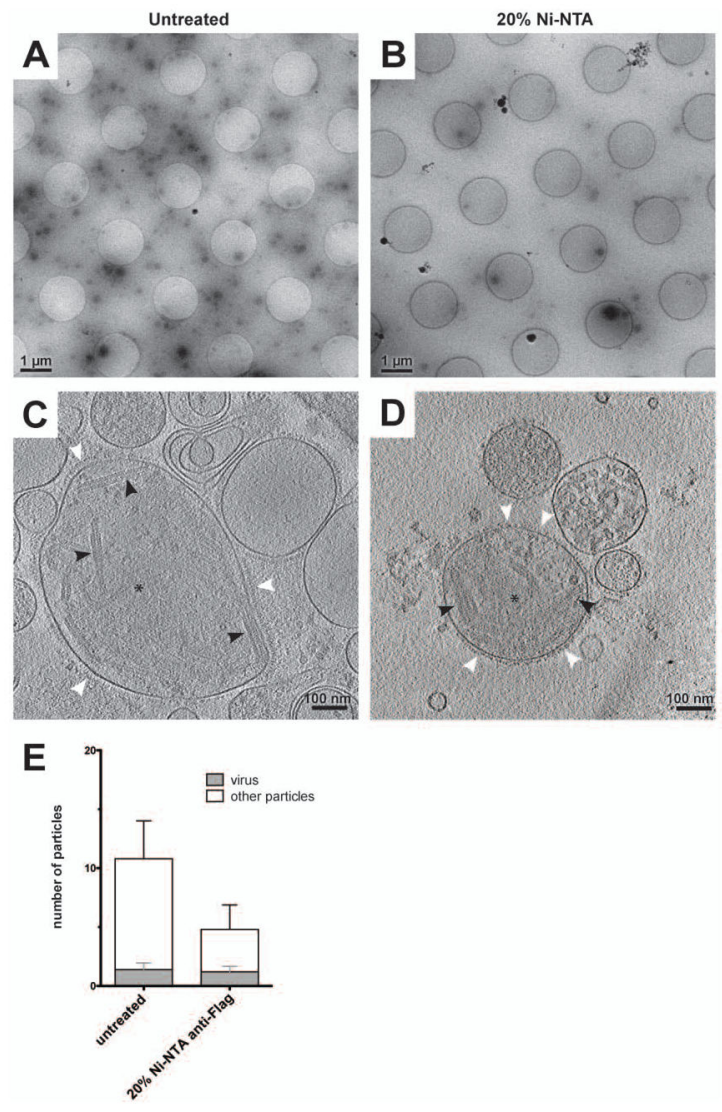

\section{Figure 2.}

Cryo-EM images and cryo-ET reconstructions of recMeV-H-Edm $3 x$ Flag particles. (A) CryoEM images of recMeV-H-Edm $3 x$ Flag particles applied to a standard cryo-EM grid. (B) recMeV-H-Edm $3 \times$ Flag particles captured on a $20 \%$ Ni-NTA cryo-affinity grid with an antiFlag antibody (1:500). (C) $7.6 \mathrm{~nm}$ slices through reconstructions of recMeV-H-Edm $3 \times$ Flag particles on standard cryo-EM grids. (D) $7.6 \mathrm{~nm}$ slices through reconstructions of recMeVH-Edm $3 x$ Flag particles on a $20 \%$ Ni-NTA cryo-affinity grid with an anti-Flag antibody (1:500). (C and D) Black asterisks denote recMeV-H-Edm $3 \times$ Flag particles, white arrowheads point to the surface glycoproteins, and black arrowheads mark the RNP. (E) Measurement of recMeV-H-Edm 3 xFlag (grey) and other (white) particles present on untreated and AG cryoEM grids. Untreated EM grids adsorbed more particles of all types. The $20 \%$ Ni-NTA AG recruited recMeV-H-Edm $3 \times$ Flag particles to the cryo-EM grid surface and reduced the quantity of other particles. Scale bars are $1 \mathrm{um}(\mathrm{A}$ and B) and $100 \mathrm{~nm}(\mathrm{C}$ and D). 

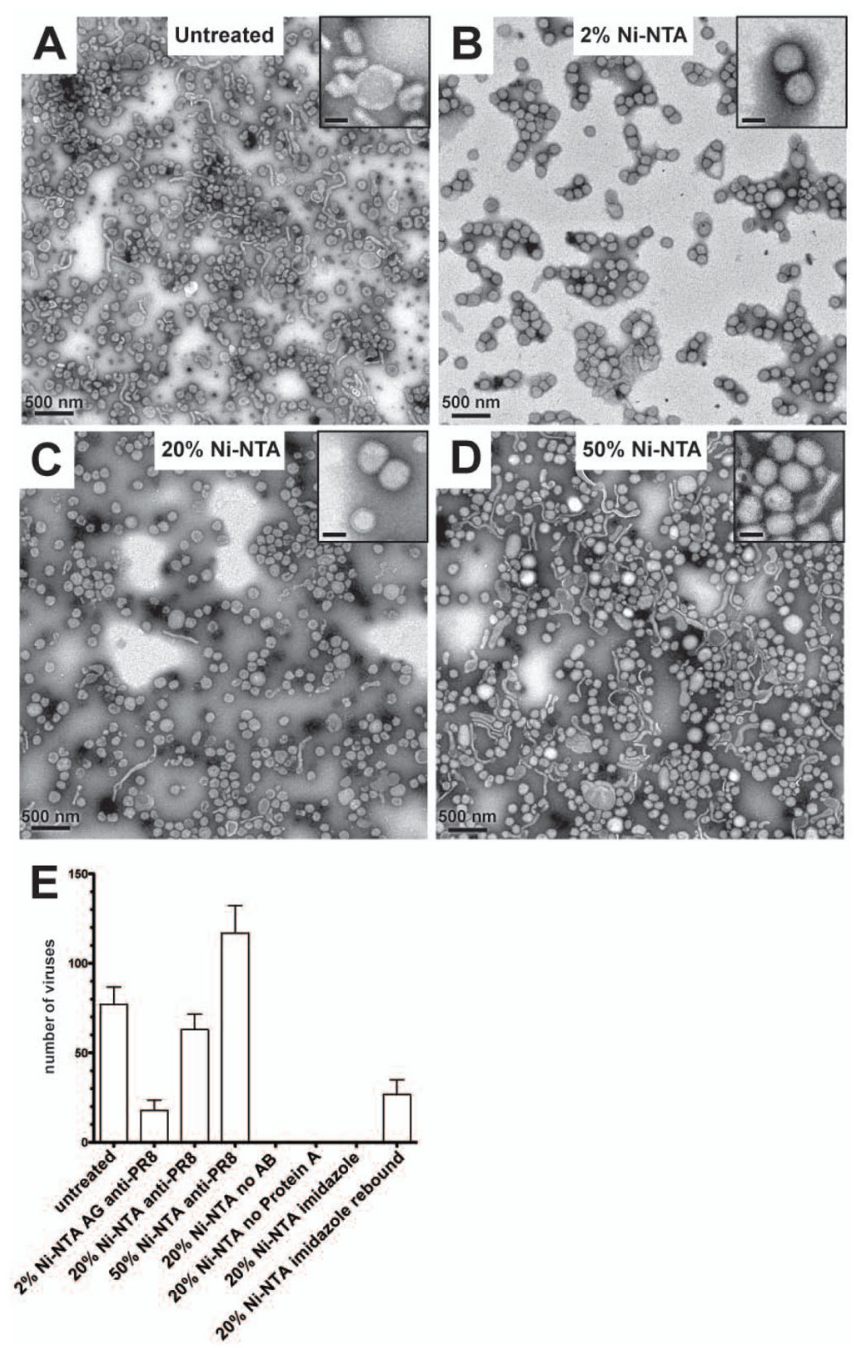

Figure 3.

TEM images of negatively stained influenza A/PR8 7:1. (A) Influenza A/PR8 7:1 particles from the resuspended pellet. (B) Influenza A/PR8 7:1 particles captured on 2\% Ni-NTA containing affinity grids using an anti-PR8 polyclonal antibody (1:500). (C) Influenza A/PR8 7:1 particles captured on 20\% Ni-NTA containing affinity grids using an anti-PR8 polyclonal antibody (1:500). (D) Influenza A/PR8 particles captured on 50\% Ni-NTA containing affinity grids using an anti-PR8 polyclonal antibody (1:500). (E) Accounting of the number of influenza A/PR8 7:1 particles adsorbed to untreated and AG EM grids. The AGs recruited influenza A/PR8 7:1 particles in a manner that was proportional to the percentage of the Ni-NTA present in the lipid monolayer. AG prepared without the anti-PR8 polyclonal antibody or the Protein-A-His did not recruit particles to the grid surface. Treatment of the AG with $600 \mathrm{mM}$ imidazole resulted in the elution of particles previously bound to the AG surface. Influenza A/PR8 7:1 particles were able to bind to the imidazoletreated surface. Images were acquired at $4 \mathrm{kx}$ and $12 \mathrm{kx}$ (insets) magnifications. Scale bars are $500 \mathrm{~nm}$ and $100 \mathrm{~nm}$ (insets). 

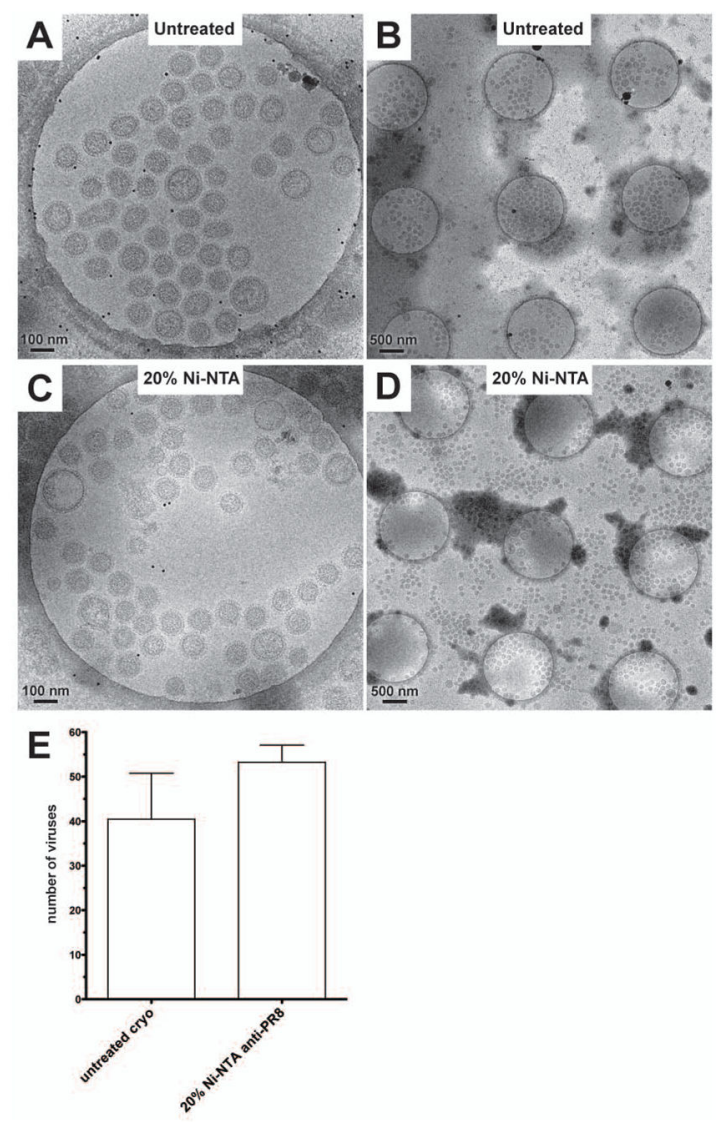

\section{Figure 4.}

Cryo-EM images of influenza A/PR8 7:1. (A and B) Cryo-EM images of influenza A/PR8 particles applied to a standard cryo-EM grid. (C and D) Cryo-EM images of influenza A/PR8 7:1 particles captured on a $20 \%$ Ni-NTA cryo-affinity grid with an anti-PR8 polyclonal antibody (1:100). (E) Measurement of influenza A/PR8 7:1 particles present on untreated and AG cryo-EM grids. Untreated EM grids adsorbed fewer influenza A/PR8 7:1 particles than the $20 \%$ Ni-NTA AG cryo-EM grid. Images were acquired at $30 \mathrm{kx}$ (A and C) and 4kx (B and D) magnifications. Scale bars are $100 \mathrm{~nm}$ (A and C) and $500 \mathrm{~nm}(\mathrm{~B}$ and D). 

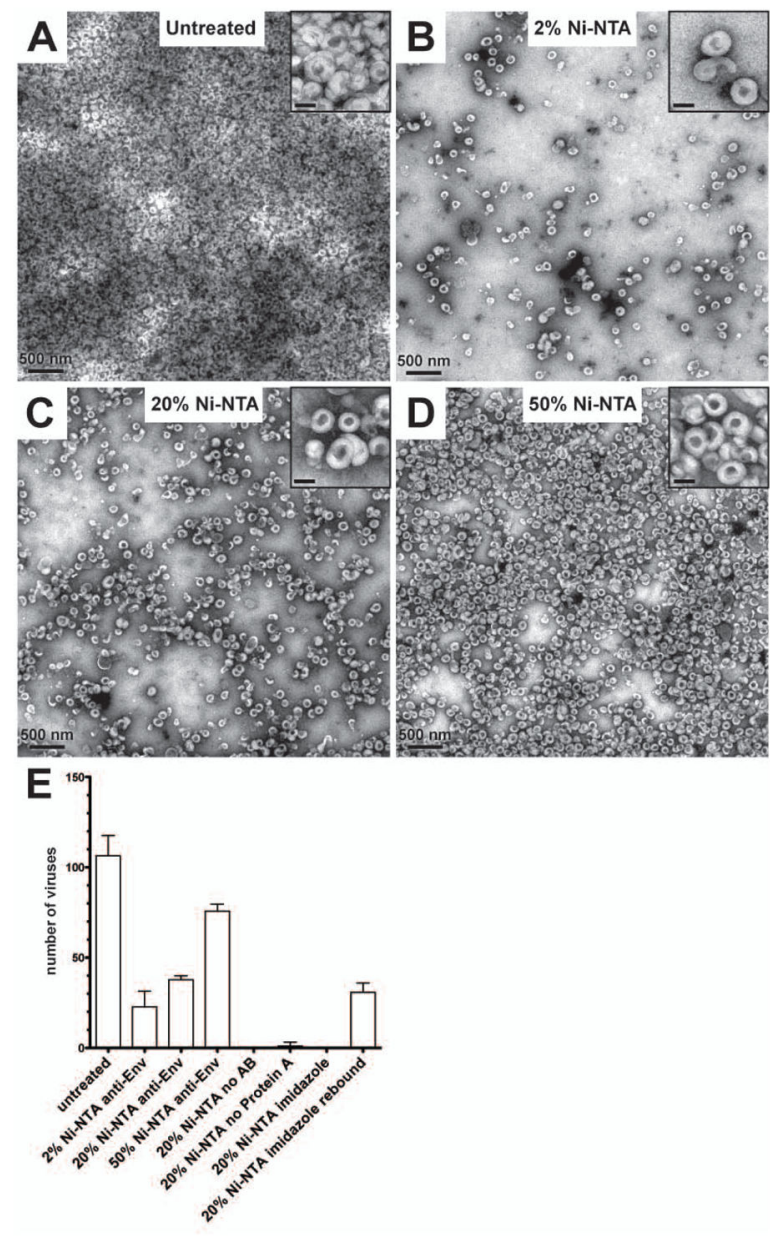

Figure 5.

TEM images of negatively stained HIV CD84 VLPs. (A) HIV CD84 VLPs from the resuspended pellet. (B) HIV CD84 VLPs captured on 2\% Ni-NTA containing affinity grids using an anti-Env polyclonal antibody (1:100). (C) HIV CD84 VLPs captured on 20\% NiNTA containing affinity grids using an anti-Env polyclonal antibody (1:100). (D) HIV CD84 VLPs captured on 50\% Ni-NTA containing affinity grids using an anti-Env polyclonal antibody (1:100). (E) The number of HIV CD84 VLPs on untreated and AG EM grids. Untreated EM grids adsorbed more total VLPs than the three AGs. The AGs retrieved HIV CD84 VLPs proportionally to the percentage of the Ni-NTA present in the lipid monolayer. AG prepared without the anti-Env antibody or the protein A did not recruit VLPs to the grid surface. Treatment of the AG with $600 \mathrm{mM}$ imidazole resulted in the elution of VLPs previously bound to the AG surface. HIV CD84 VLPs could be rebound to the AG surface after its treatment with imidazole. Images were acquired at $4 \mathrm{kx}$ and $12 \mathrm{kx}$ (insets) magnifications. Scale bars are $500 \mathrm{~nm}$ and $100 \mathrm{~nm}$ (insets). 

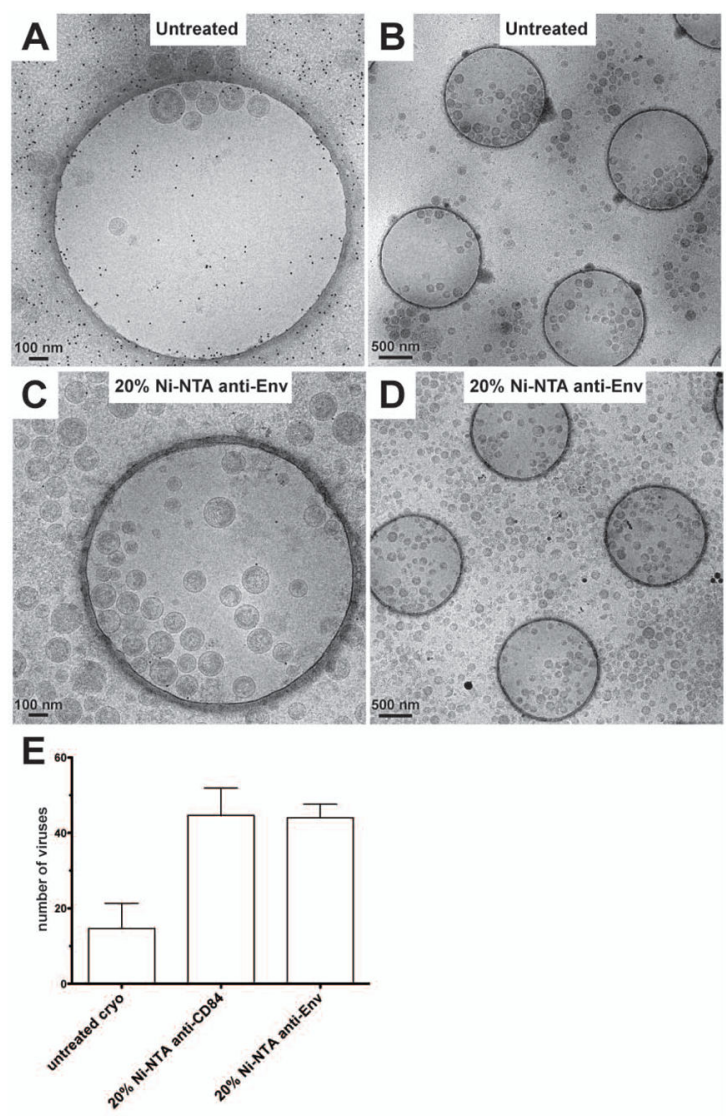

Figure 6.

Cryo-EM images of HIV CD84 VLPs. (A and B) Cryo-EM images of HIV CD84 VLPs applied to a standard, untreated cryo-EM grid. (C and D) Cryo-EM images of HIV CD84 VLP particles captured on a $20 \%$ Ni-NTA cryo-affinity grid with an anti-Env polyclonal antibody (1:100). (E) The amount of HIV CD84 VLPS on untreated and AG cryo-EM grids. Untreated EM grids adsorbed fewer HIV CD84 VLPs than the 20\% Ni-NTA AG cryo-EM grid. Images were acquired at 30kx (A and C) and 4kx (B and D) magnifications. Scale bars are $100 \mathrm{~nm}$ (A and C) and $500 \mathrm{~nm}(\mathrm{~B}$ and $\mathrm{D})$. 